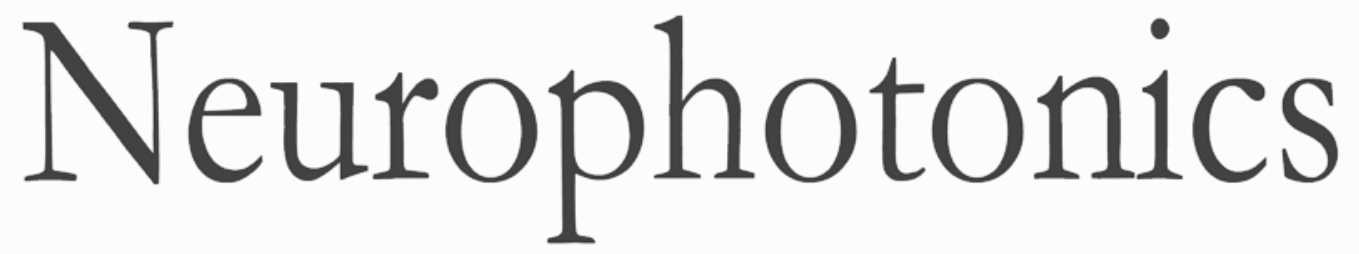

\title{
Review of imaging network activities in developing rodent cerebral cortex in vivo
}

Heiko J. Luhmann 


\title{
Review of imaging network activities in developing rodent cerebral cortex in vivo
}

\author{
Heiko J. Luhmann* \\ University Medical Center of the Johannes Gutenberg University Mainz, Institute of Physiology, Duesbergweg 6, 55128 Mainz, Germany
}

\begin{abstract}
The combination of voltage-sensitive dye imaging (VSDI) with multielectrode array (MEA) recordings in the rodent cerebral cortex in vivo allows the simultaneous analysis of large-scale network interactions and electrophysiological single-unit recordings. Using this approach, distinct patterns of spontaneous and sensoryevoked activity can be recorded in the primary somatosensory (S1) and motor cortex (M1) of newborn rats. Already at the day of birth, gamma oscillations and spindle bursts in the barrel cortex synchronize the activity of a local columnar ensemble, thereby generating an early topographic representation of the sensory periphery. During the first postnatal week, both cortical activity patterns undergo developmental changes in their spatiotemporal properties and spread into neighboring cortical columns. Simultaneous VSDI and MEA recordings in $\mathrm{S} 1$ and $\mathrm{M} 1$ demonstrate that the immature motor cortex receives information from the somatosensory system and that M1 may trigger movements of the periphery, which subsequently evoke gamma oscillations and spindle bursts in S1. These early activity patterns not only play an important role in the development of the cortical columnar architecture, they also control the ratio of surviving versus dying neurons in an activity-dependent manner, making these processes most vulnerable to pathophysiological disturbances during early developmental stages. () 2016 Society of Photo-Optical Instrumentation Engineers (SPIE) [DOI: 10.1117/1.NPh.4.3.031202]
\end{abstract}

Keywords: voltage-sensitive dye imaging; neocortex; development; rat; in vivo.

Paper 16057SSVR received Aug. 16, 2016; accepted for publication Oct. 19, 2016; published online Nov. $23,2016$.

\section{Introduction}

The very immature cerebral cortex reveals a surprisingly rich repertoire of spontaneous and sensory-evoked neuronal network activity (for review, see Refs. 1-3). Already at the day of birth (postnatal day [P] 1), sensory neocortical areas of rodents show distinct patterns of synchronized network activity: so-called spindle bursts (for review, see Ref. 4) and gamma oscillations (for review, see Ref. 5). Spindle bursts are local network oscillations in a frequency range of 10 to $20 \mathrm{~Hz}$ and with a duration of 0.5 to $3 \mathrm{~s}$. These events occur spontaneously every $\sim 10 \mathrm{~s}$ or can be elicited by a single stimulus of the sensory periphery. So far, spindle bursts have been demonstrated in the visual cortex $x^{6,7}$ and somatosensory cortex. ${ }^{8-10}$ Gamma oscillations with a frequency of 30 to $40 \mathrm{~Hz}$ and a duration of 100 to $300 \mathrm{~ms}$ can also be evoked by sensory stimulation or appear spontaneously every 10 to $30 \mathrm{~s}^{10,11}$ Gamma oscillations are restricted to local functional columns in the barrel cortex of newborn rats in vivo, at a stage before all six cortical layers have been formed and before anatomical columns can be identified. ${ }^{12}$

At a developmental stage, when the human cerebral cortex can be compared with that of a newborn rodent, similar spontaneous and sensory-evoked activity patterns, so-called "delta brushes," have been recorded with an electroencephalogram (EEG) in preterm human babies (for review, see Refs. 2 and 13). Delta brushes are high-amplitude waves in delta rhythm superimposed with rapid (>8 Hz) oscillations (the "brush"), which can be observed in all neocortical areas of preterm infants between postmenstrual week 28 to around birth. As in newborn

*Address all correspondence to: Heiko J. Luhmann, E-mail: luhmann@ uni-mainz.de rodents, this early cortical activity in premature infants occurs spontaneously as well as upon sensory stimulation. ${ }^{14,15}$

It has been demonstrated in the barrel cortex of rodents that the experimental elimination of spontaneous and sensoryevoked spindle bursts by selective removal of the subplate prevents the development of the characteristic whisker-related columnar architecture, ${ }^{16}$ indicating that this early cortical activity pattern and the subplate play an important role in cortical maturation (for review, see Ref. 4). EEG and magnetic resonance imaging measurements in premature human babies have demonstrated that increased levels of neocortical activity are associated with better brain growth ${ }^{17}$ and that the dynamics of this EEG activity predict the later mental development of the infant. $^{18}$

This review aims to provide an overview on the spatiotemporal properties and dynamics of neuronal network activity in the developing rodent cerebral cortex in vivo.

\section{VSDI as a Tool to Study Neocortical Network Activity}

Voltage-sensitive dye imaging (VSDI) is a very powerful technique to study, with excellent spatial and temporal resolution, local and large-scale neuronal activity patterns in the cerebral cortex of various mammalian species from rodents to primates (for review, see Refs. 19-21). In vivo, VSDI has been successfully applied to study the spatiotemporal dynamics of neocortical network activity in anesthetized, behaving head-fixed, and behaving freely moving mammals (for review, see Refs. 22 and 23). However, in vivo imaging also has some limitations because recordings from lower neocortical layers are difficult to gain unless a microendoscope is implanted. ${ }^{24,25}$ To overcome this 


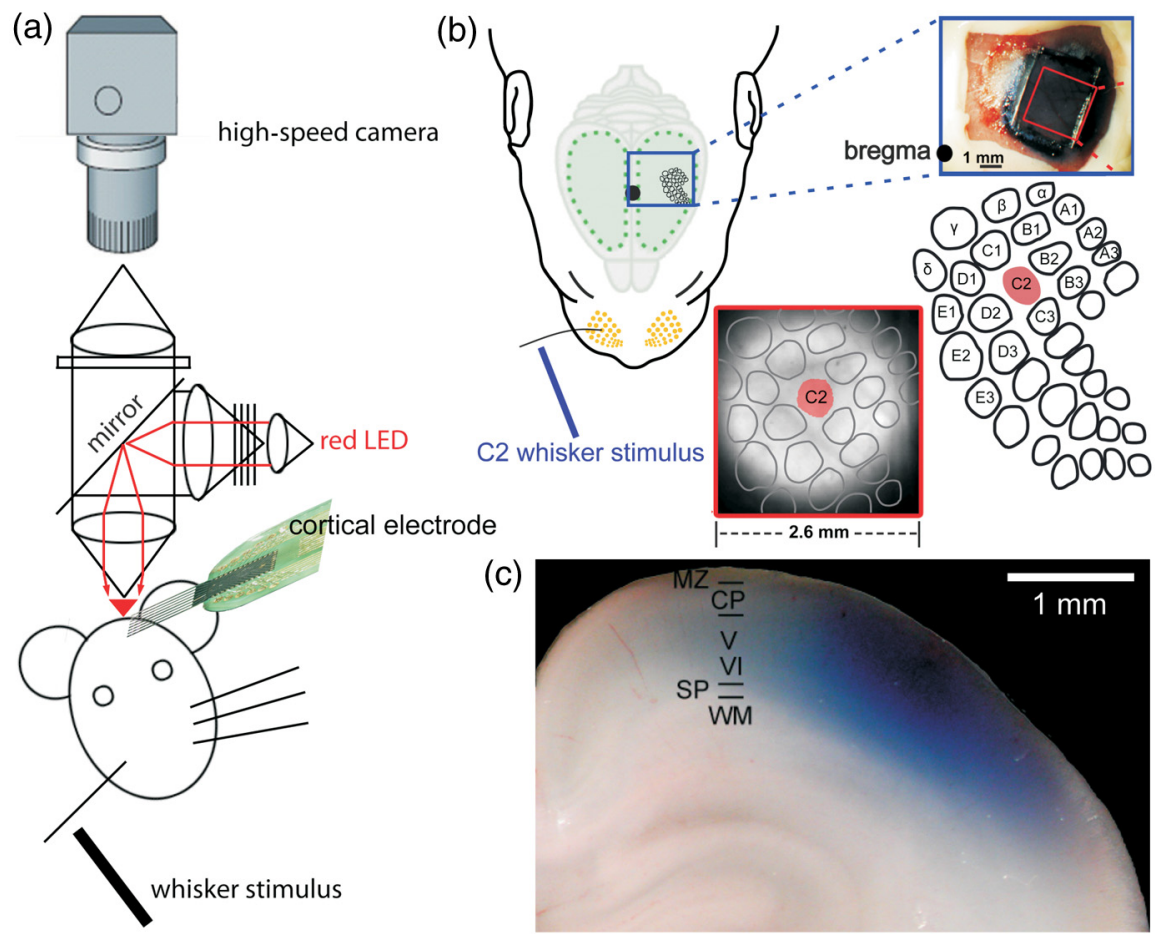

Fig. 1 Simultaneous VSDI with RH1691 and MEA recordings in barrel cortex of newborn rat in vivo. (a) Schematic diagram of experimental setup combining single-whisker stimulation, VSDI, and cortical multielectrode recordings. (b) Selective mechanical stimulation of whisker C2 (left) elicits a local VSD response in the representation of the $\mathrm{C} 2$ whisker in the contralateral cortical barrel field (lower panels). Barrel field map is superimposed onto the fluorescence image. Upper-right photograph shows the view from the top on parietal cortex stained with RH1691. (c) Photograph of coronal section from parietal cortex of a P0 rat illustrating penetration and staining pattern of $\mathrm{RH} 1691$ (blue). Modified with permission from Ref. 12.

problem, we combine VSDI with multichannel extracellular recordings employing multielectrode arrays (MEAs) reaching infragranular layers in barrel cortex of adult ${ }^{26,27}$ and newborn rats. ${ }^{10,12,28}$

We combine VSDI and MEA recordings in the cerebral cortex of newborn rodents in vivo to gain a better understanding of the function and mechanisms underlying the generation of early neocortical activity patterns. Although VSDI offers the advantage that network activity can be monitored in vivo over large neocortical territories ${ }^{29,30}$ (for review, see Ref. 21), MEAs allow extracellular recordings from deeper structures with high temporal and cellular resolution ${ }^{27,31,32}$ (for review, see Ref. 33). Thus, VSDI and MEA recordings are complementary techniques that, in combination, provide important insights into the function of local and global network activities in the developing cerebral cortex.

\section{Combining VSDI with Multichannel Extracellular Recordings In Vivo}

In vivo recordings are obtained in the primary somatosensory cortex (S1) and primary motor cortex (M1) of P0-P7 Wistar rats using experimental protocols as described in detail before $^{10}$ (Fig. 1). The animals are lightly anesthetized by intraperitoneal urethane $(<0.2 \mathrm{~g} / \mathrm{kg}$, Sigma-Aldrich, Taufkirchen, Germany) and the animal's head is fixed with dental cement on the occipital bones via an aluminum holder into a stereotaxic apparatus. The skull above S1 and M1 is thinned on the left hemisphere using a miniature drill until the residual bone, but not the dura mater, can be carefully removed. The VSD
RH1691 (Optical Imaging, Rehovot, Israel) is dissolved at $1 \mathrm{mg} / \mathrm{ml}$ in saline solution and topically applied to the cortical surface for $20 \mathrm{~min}$ [Fig. 1(b)]. In newborn rodents, all neocortical layers from the marginal zone (MZ)/layer (L) I to the subplate (SP) are stained [Fig. 1(c)]. The cortex is covered with $1 \%$ low-melting agarose and a cover slip is placed on top to stabilize the tissue. Excitation light from a red light-emitting diode (LED) (MRLED $625 \mathrm{~nm}$, Thorlabs GmbH, Dachau, Germany) is band-pass filtered $(630 / 30 \mathrm{~nm})$, reflected by a 650-nm dichroic mirror, and focused onto the cortical surface with a 25-mm Navitar lens (Stemmer Imaging, Puchheim, Germany) [Fig. 1(a)]. The emitted fluorescence is collected via the same optical pathway, long-pass filtered $(660 \mathrm{~nm})$, and focused via another 25-mm Navitar lens onto the chip of a MiCam Ultima L high-speed camera (Scimedia, Costa Mesa, California). This tandem-type macroscope design ${ }^{34}$ results in a $1 \times$ magnification, and every pixel of this camera collects light from a neocortical region of $26 \times 26 \mu \mathrm{m}^{2}$. Signals are recorded at a sampling frequency of $500 \mathrm{~Hz}$, and the signalto-noise ratio is improved using $5 \times 5$ pixel spatial binning followed by $60-\mathrm{Hz}$ low-pass filtering. Bleaching of fluorescence is corrected by subtraction of a best-fit double-exponential or fifth-degree polynomial. The normalized change of fluorescence intensity $\left(\Delta F / F_{0}\right)$ is calculated as the change of fluorescence intensity $(\Delta F)$ in each pixel divided by the initial fluorescence intensity $\left(F_{0}\right)$ from the same pixel. Fluorescent changes of $>0.2 \%$ are considered responses.

Local field potentials (LFPs) and multiple-unit activity (MUA) are recorded (1) in the barrel cortex with an 8-shank 
(a)
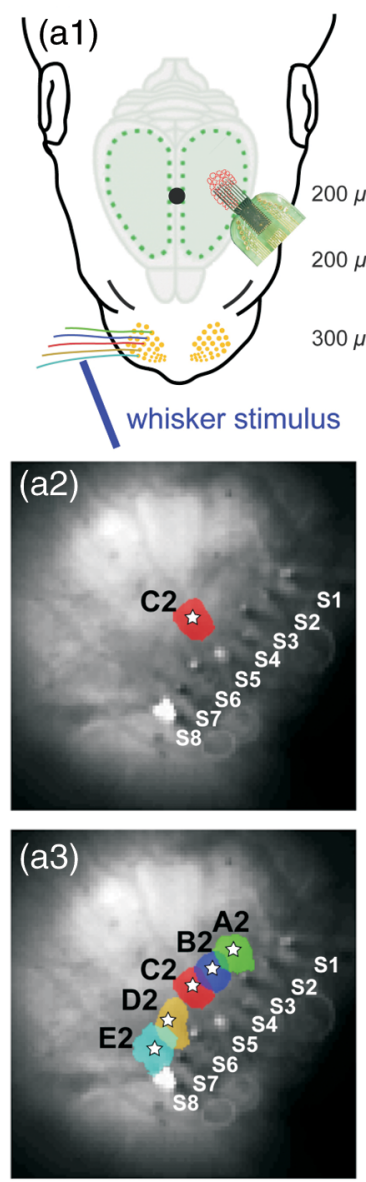

(b)

(b1)
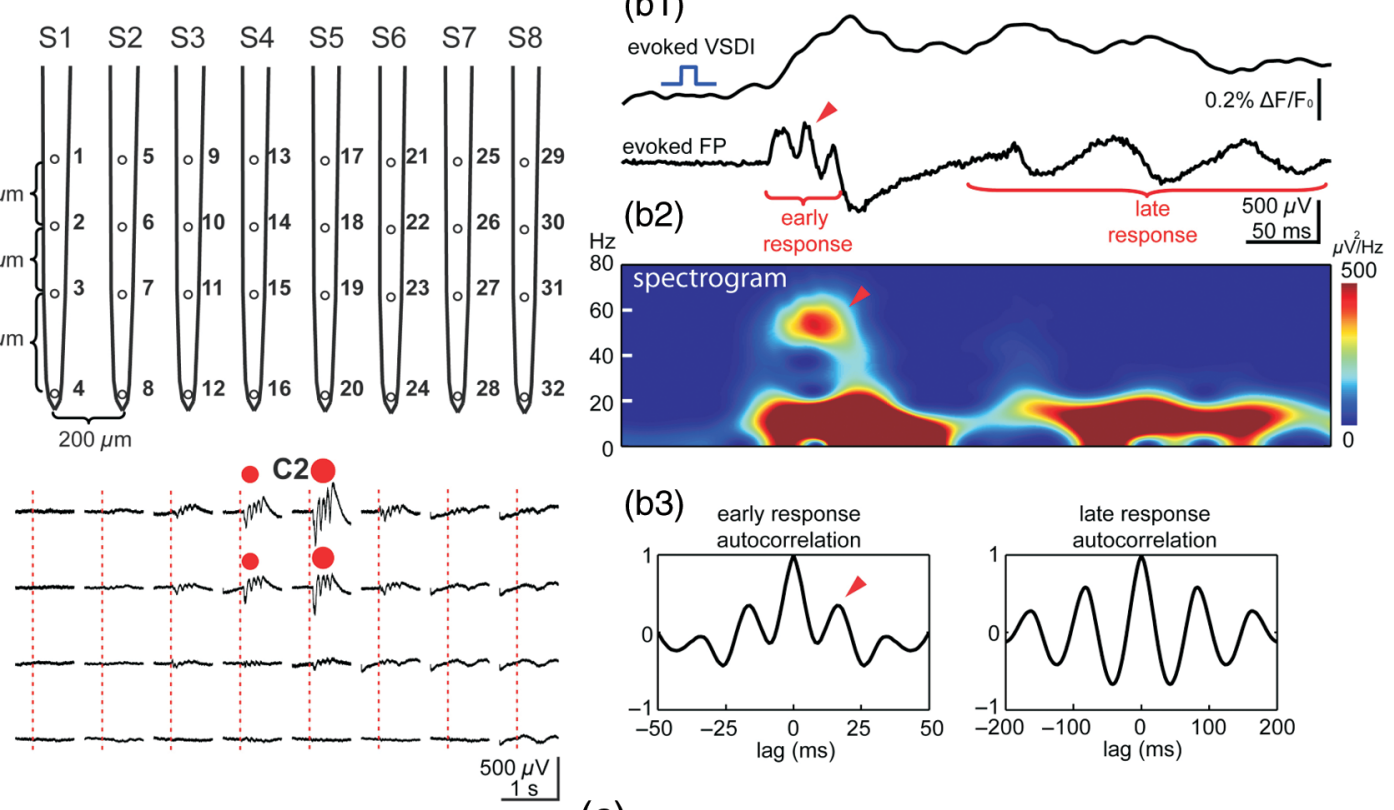

(b3)
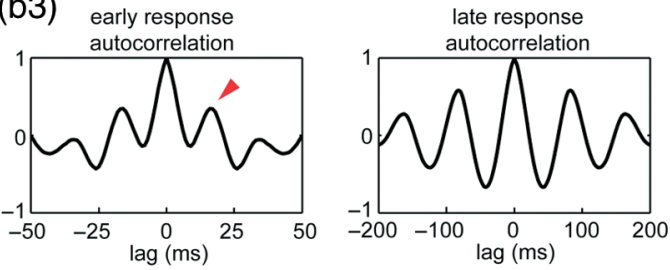

(c)

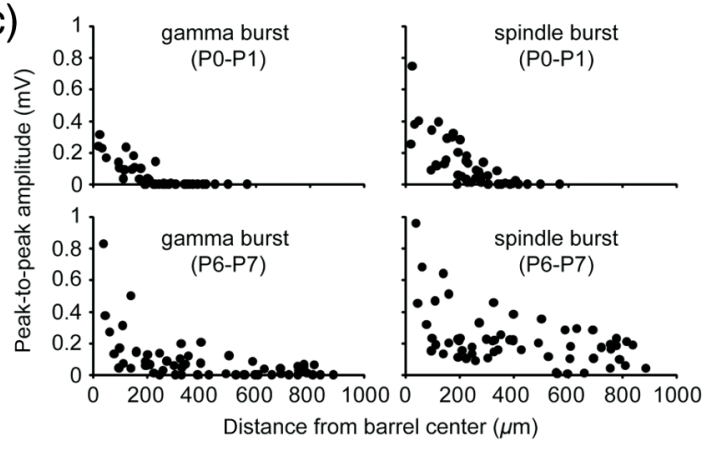

Fig. 2 Combined VSDI and large-scale multielectrode recordings reveal spatiotemporal properties of sensory-evoked activity in newborn rat barrel cortex in vivo. (a1) Experimental setup combining mechanical single-whisker stimulation, VSDI, and cortical 32-channel recordings using an 8-shank electrode (S1-S8, spacing $200 \mu \mathrm{m}$ ). (a2) Selective stimulation of whisker C2 in a P1 rat elicits a local VSD (left) and local electrophysiological (right) responses in the cortical C2 barrel. Note oscillatory response pattern restricted to the upper cortical network with a horizontal extent of $\sim 200 \mu \mathrm{m}$. (a3) Color-coded map of the evoked VSD (left) and electrophysiological (right) responses to stimulation of single-whisker A2-E2. Size of the color-coded circle depicts the peak amplitude of electrophysiological response. (b1) Simultaneously recorded VSD and LFP responses in cortical E2 barrel upon stimulation whisker $\mathrm{E} 2$ in a P1 rat. Note fast early (gamma burst) and slower late oscillatory responses (spindle burst). (b2) Spectrogram of the LFP response and (b3) autocorrelograms of early and late LFP responses reveals the frequency of the network oscillations. (c) Relationship between LFP amplitude and distance from the barrel center for sensory-evoked gamma bursts (left) and spindle bursts (right) in P0-P1 (upper panels, $n=44$ recordings in four animals) and P6-P7 rats (lower panels, $n=59$ recordings in five animals). Reproduced with permission from Ref. 12.

32-channel Michigan-type electrode (1 to $2 \mathrm{M} \Omega$, NeuroNexus Technologies, Ann Arbor, Michigan) [Fig. 2(a1)] or (2) simultaneously in the forepaw representation of S1 and M1 with two 4-shank 16-channel Michigan electrodes [Fig. 3(a)] as described previously. ${ }^{12,35}$ For recordings in barrel cortex, VSDI responses to single mechanical stimulation of whisker A2-E2 are used to identify the location of the arc 2 barrel-related columns. The electrode array [spacing 200 or $300 \mu \mathrm{m}$ in vertical direction and $200 \mu \mathrm{m}$ in horizontal direction; see Fig. 2(a1)] is then inserted at an angle of $\sim 35 \mathrm{deg}$ into the barrel cortex representing the whiskers of arc 2 [Fig. 2(a3)]. For simultaneous recordings in $\mathrm{S} 1$ and M1, one electrode array [spacing $50 \mu \mathrm{m}$ in the vertical direction and $125 \mu \mathrm{m}$ in the horizontal direction; see Fig. 3(a4)] is inserted into the center of the forepaw representation of S1 and M1 as identified by VSDI [Fig. 3(b1)]. LFPs and MUA are recorded at a sampling rate of $20 \mathrm{kHz}$. The animals are kept at a constant body temperature of $37^{\circ} \mathrm{C}$, and recordings can be obtained for up to $7 \mathrm{~h}$.

\section{Sensory-Evoked and Spontaneous Local Activity in Newborn Rat Barrel Cortex}

A defined mechanical stimulation of a single whisker elicits reliable VSD and LFP responses in the barrel cortex of the contralateral hemisphere consisting of an initial gamma oscillation followed by a spindle burst [Fig. 2(b1)]. This observation demonstrates that both types of electrophysiological activity patterns contribute to the optical signal. The wavelet spectrogram 
(a) (a1)

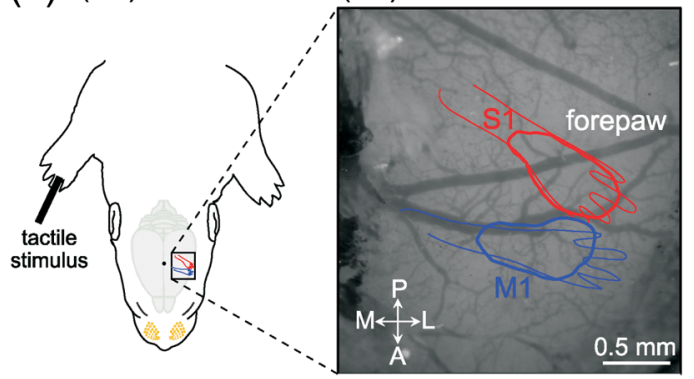

(b)

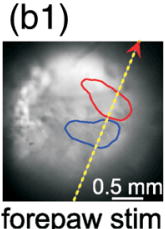

(a2)

)

forepaw stim

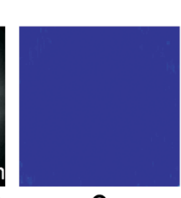

0

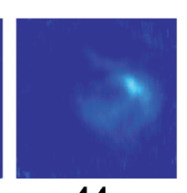

44

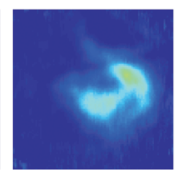

52 (a3)

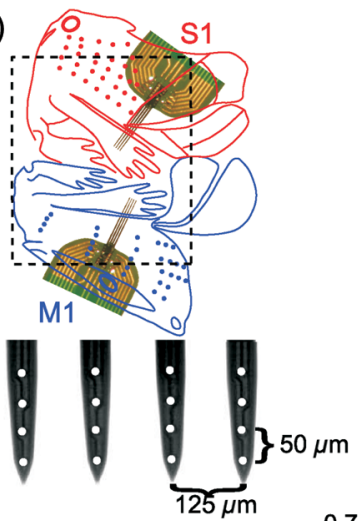

(b2)
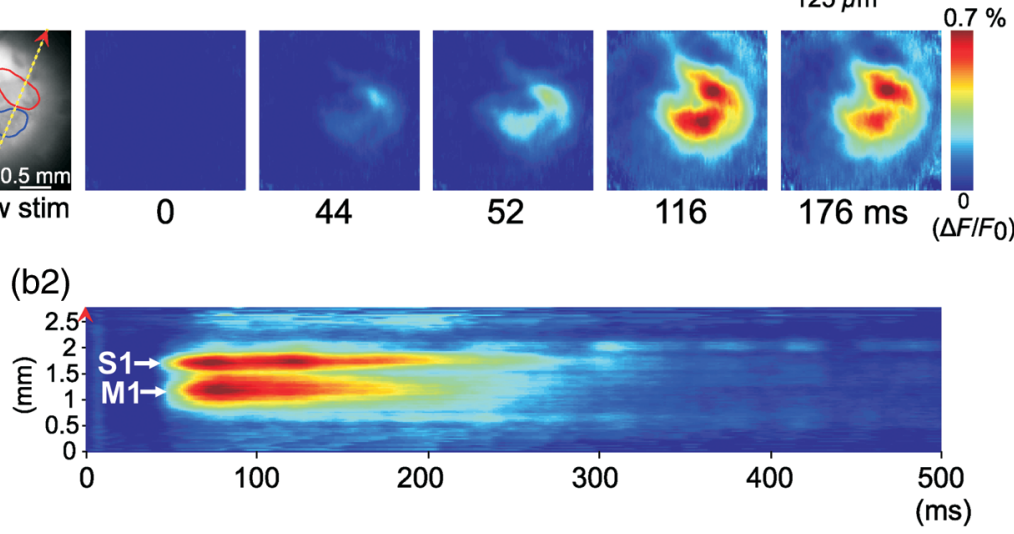

(c)

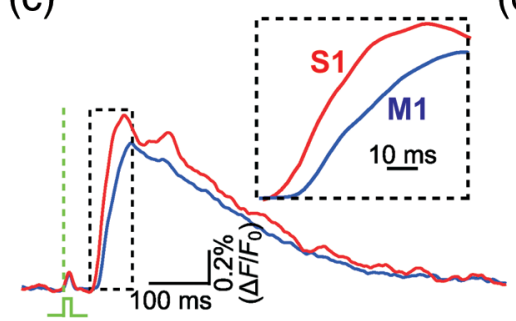

forepaw stim (d)

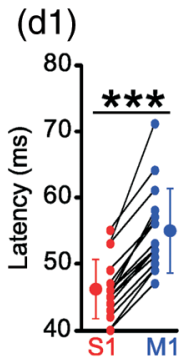

(d2)

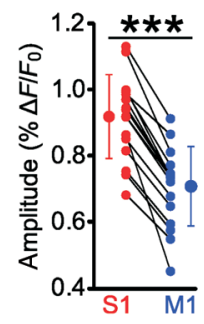

(d3)

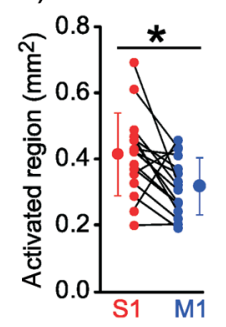

Fig. 3 Simultaneous VSDI and MEA recordings in primary somatosensory cortex (S1, red) and primary motor cortex (M1, blue) of newborn rat in vivo. (a1 and a2) Schematic illustration of experimental setup combining selective mechanical stimulation of the forepaw and simultaneous VSDI recordings in the forepaw representation of S1 and M1. A, anterior; L, lateral; P, posterior; M, medial. (a3 and a4) Body representation in S1 and M1 cortex with cortical region shown in (a2) (black dashed square) and localization of the $4 \times 4$ MEA in the forepaw representation of S1 and M1. (b1) Same cortical region as in (a2) with VSD responses in S1 and M1 to a single mechanical stimulus of the right forepaw $(t=0 \mathrm{~ms})$ at different time points poststimulus. (b2) VSD response was scanned along the yellow dashed line (indicated on the left image of b1) from 0 to $500 \mathrm{~ms}$ poststimulus. Note the two clear distinct responses in S1 and M1. (c) Average of 10 VSD responses in S1 (red) and M1 (blue) to single forepaw stimulation. Time point of mechanical stimulation of the forepaw at $t=0 \mathrm{~ms}$ (green dashed line). Inset shows a representative response at an expanded time scale. Note that VSD response is S1 and precedes response in M1 by $10 \mathrm{~ms}$. (d1) Average onset latency, (d2) maximal amplitude, and (d3) size of activated cortical region of forepaw-evoked VSD response ( $n=16$ P3-P5 rats). Responses in S1 are significantly faster and larger compared with responses in M1. Small symbols connected by black lines represent individual animals. Larger symbols express mean \pm SD. Significant differences between S1 and M1 at $p<0.001\left(^{* \star *}\right)$ and $p<0.05\left(^{*}\right)$ as tested with Mann-Whitney-Wilcoxon test. Modified from Ref. 35.

[Fig. 2(b2)] and the autocorrelation analyses [Fig. 2(b3)] illustrate the characteristic gamma oscillation of the early response and the $\sim 10 \mathrm{~Hz}$ frequency in the subsequent spindle burst. In P0-P1 rats, both the VSDI and LFP responses to single-whisker stimulation are highly localized and at that age do not spread to neighboring cortical columns [Fig. 2(a2)]. The topographic representation of the five single-arc 2 whiskers A2-E2 in the contralateral barrel cortex can be revealed by simultaneous
VSDI and MEA recordings. After appropriate positioning of all 8-shank recording electrodes (S1-S8) into the whisker-related columns of the whisker A2-E2, single-whisker stimulation evokes a local VSDI and corresponding local electrophysiological response that is organized in a topographical manner already in $\mathrm{P} 0$ animals [Fig. 2(a3)], ${ }^{12}$ by the latest at P2/P3. ${ }^{36}$

Plotting the peak-to-peak amplitude of the evoked electrophysiological response against its distance to the center of 
the barrel as identified by VSDI shows that gamma bursts in P0-P1 rats are confined to an area $\sim 400 \mu \mathrm{m}$ in diameter, whereas spindle bursts at that age cover a slightly larger area $\sim 600 \mu \mathrm{m}$ in diameter [upper panels in Fig. 2(c)]. In P6-P7 rats, the spatial extent of the sensory-evoked response gets larger and the activity spreads into neighboring cortical columns [lower panels in Fig. 2(c)]. Analyzing the cortical VSDI responses to $\mathrm{C} 2$ whisker stimulation in more detail reveals significant age-dependent differences in their spatial extent, onset latency, and duration. Whereas in P0 rats the evoked response is spatially restricted to an average cortical region $\sim 320 \mu \mathrm{m}$ in diameter, the same stimulus in P7 rats elicits an average response $>1400 \mu \mathrm{m}$ in diameter. ${ }^{12}$ This age-dependent increase in the size of the activated cortical network is accompanied by (1) a developmental decrease in the average onset latency of the cortical response from $\sim 90 \mathrm{~ms}$ at $\mathrm{P} 0$ to $\sim 35 \mathrm{~ms}$ at P7 and (2) a decrease in the mean response duration at half-maximal amplitude from $\sim 300 \mathrm{~ms}$ in P0-P1 rats to $\sim 170 \mathrm{~ms}$ at P7. During subsequent development, sensory-evoked responses undergo further changes in their spatiotemporal profile. Borgdorff et al. ${ }^{37}$ demonstrated in mouse barrel cortex that single-whisker deflection in P7-P12 animals evoked a smaller, slower, and more localized response compared with P13-P21 mice.

Spontaneous events recorded with VSDI in P0-P1 rats occur every $\sim 5 \mathrm{~s}$ and have similar spatiotemporal properties as the evoked cortical responses at this age. At this age, spontaneous events are confined to an average region of $\sim 390 \mu \mathrm{m}$ in diameter and show a duration at half-maximal amplitude of $\sim 250 \mathrm{~ms}$. These observations demonstrate that spontaneous events have very similar spatiotemporal properties as the sensory-evoked responses recorded with VSDI. Interestingly, when the spontaneous events recorded in $\mathrm{P} 0-\mathrm{P} 1$ rats are superimposed on the predicted barrel field map of each individual animal, the large majority of these spontaneous events are restricted to 1 or 2 "prebarrel" related columns. ${ }^{12}$ In contrast, in P6-P7 rats, spontaneous events occur twice as often (every $2.6 \mathrm{~s}$ ) compared with P0-P1 pups, which have a shorter average duration at half-maximal amplitude ( $184 \mathrm{~ms}$ ) and more often spread into neighboring cortical columns, as previously observed in the barrel cortex of anesthetized adult rats ${ }^{38}$ and freely moving mice. $^{39}$

Spontaneous activity patterns, which are highly synchronous within local clusters of supragranular neurons, have also been observed with two-photon calcium imaging in the barrel cortex of unanesthetized P4 mice. ${ }^{40}$ In the same study, a transition in the pattern of spontaneous activity from this synchronized to a more desynchronized pattern has been demonstrated. Using three-dimensional two-photon calcium imaging and simultaneous extracellular recordings, clusters of neurons coactively synchronized in spindle bursts and organized in a columnlike manner have been identified in the visual cortex of P3$\mathrm{P} 4$ mice in vivo. ${ }^{41,42}$ These data, obtained in different neocortical areas of newborn rodents in vivo, indicate that spontaneous active neurons self-organize into discrete columnar networks during the earliest stages of development, forming the structural and functional template for the maturation of the columnar architecture.

Using simultaneous MEA recordings in the thalamic ventral posterior medial (VPM) nucleus and barrel cortex of P0-P1 rats in vivo, we have shown that spontaneously occurring and singlewhisker-evoked cortical activity correlates with local thalamic activity, ${ }^{12}$ indicating that the thalamus plays an important role in the generation of this early activity or its transmission to the cerebral cortex. We could also demonstrate that local electrical stimulation of a single barreloid in the VPM evokes a local cortical response in the corresponding barrel-related column, which resembles the sensory-evoked cortical response following mechanical whisker stimulation. This observation in the somatosensory system of the newborn rat is in good agreement with previous data obtained in the visual system of ferrets and cats, where an early segregation of ocular dominance columns before the thalamocortical afferents innervate L4 has been documented. ${ }^{43}$ These data further demonstrate that the basic mosaic-like structure of the cortical columnar architecture develops very early, ${ }^{44}$ as proposed in the radial unit hypothesis of cortical development. ${ }^{45,46}$ The thalamic input does show its mature cortical innervation pattern at this early age, but forms transient functional glutamatergic inputs to subplate neurons ${ }^{10,47-49}$ (for a review, see Ref. 50). At this age, the subplate plays a central role in thalamocortical information transfer and amplification of oscillatory activity ${ }^{51}$ (for a review, see Ref. 52). Removal of the subplate not only eliminates oscillatory activity in the immature cortical network, ${ }^{53}$ it also prevents the normal formation of the typical barrel field structure. ${ }^{16}$

However, the subplate may not be the network generating the gamma oscillations and spindle bursts. Experimental studies in the developing visual, auditory, and somatosensory systems have shown that the sensory periphery plays an important role in driving spontaneously occurring activity at early ages (for a recent review, see Ref. 54). Spontaneous spindle bursts in the visual cortex are correlated with retinal bursts, ${ }^{6}$ and before eye opening, $87 \%$ of the spontaneous cortical activity is generated in the retina. ${ }^{55}$ Injection of lidocaine into the whisker pad reduces the occurrence of gamma oscillations and spindle bursts by $\sim 50 \%,{ }^{10}$ supporting the hypothesis that the sensory periphery is critically involved in the generation of these early neocortical activity patterns. Although the retinal waves are intrinsically generated within the retina (for review, see Ref. 56), it is less clear how spontaneous activity is generated in the peripheral mechanosensors of the somatosensory system. In adult rodents, whisker movements are controlled by the somatosensory and motor $\operatorname{cortex}^{57,58}$ (for a review, see Ref. 59). To address the question of whether whisker movements in newborns are also controlled by the cortex, we performed simultaneous VSDI and MEA recordings in the somatosensory and motor cortex of newborn rats in vivo.

\section{Early Interaction Between the Somatosensory System and the Motor System}

Because it is rather difficult to monitor the whisker movements in a newborn rat, we instead studied the forepaw by recording its movements with a piezoelectric transducer and by mechanical stimulation using a miniature solenoid actuator. ${ }^{35}$ After identification of the forepaw representation in S1 and M1 by VSDI, one 4-shank 16-channel Michigan electrode was inserted into both cortical regions [Fig. 3(a)]. A single stimulus to the forepaw reliably evokes a local VSDI response in the contralateral $\mathrm{S} 1$ at a latency of $\sim 46 \mathrm{~ms}$ [Fig. 3(b)]. At a latency of $\sim 55 \mathrm{~ms}$, a local response in the contralateral M1 can be detected [Figs. 3(b)-3(d)]. Compared with the VSDI response in S1, the response in M1 has a significantly smaller amplitude [Fig. 3(d2)] and smaller spatial extent [Fig. 3(d3)]. These observations obtained with VSDI are confirmed by electrophysiological 
(a)

(a1)
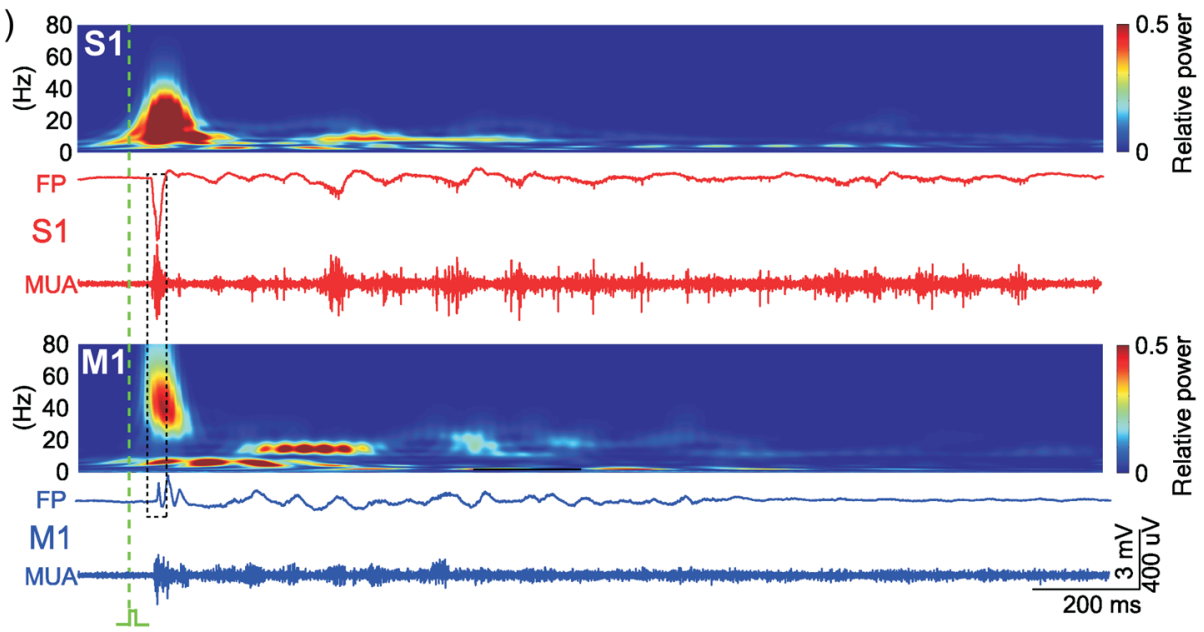

(a2)

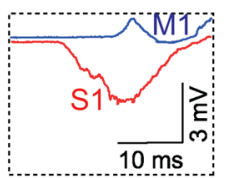

(b)

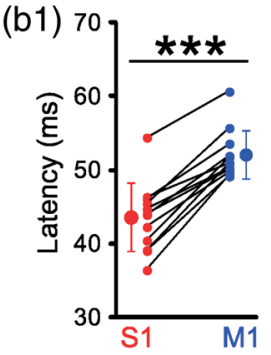

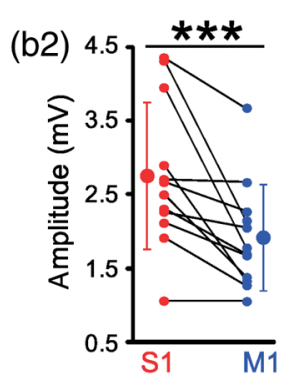

(b3)

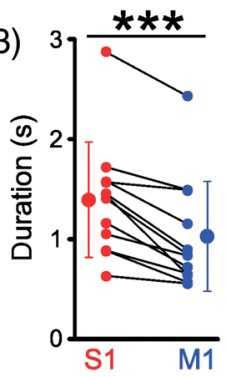

Fig. 4 Electrophysiological responses in S1 and M1 to mechanical forepaw stimulation. (a1) Burst responses in $\mathrm{S} 1$ (red) and M1 of a P4 rat. Wavelet analyses above are calculated from the unfiltered LFP recordings and MUA is high-pass filtered $(>200 \mathrm{~Hz})$. Time point of forepaw stimulation is indicated by green dashed line. (a2) Evoked response in S1 precedes response in M1. (b1) Average statistical onset latency, (b2) maximal amplitude, and (b3) duration of forepaw-evoked LFP responses $(n=12$ P3-P5 rats). Note similar properties of electrophysiological responses to VSD responses shown in Figs. 3(c) and 3(d). Small symbols connected by black lines represent the individual animals. Larger symbols express mean \pm SD. Significant differences between $\mathrm{S} 1$ and M1 at $p<0.001{ }^{(* *)}$ as tested with Mann-Whitney-Wilcoxon test. Modified from Ref. 35.

recordings using Michigan-type electrodes [Fig. 4(a)]. A single mechanical stimulus of the forepaw elicits an LFP response in the contralateral S1 at a latency of $\sim 44 \mathrm{~ms}$ and in M1 at $\sim 52 \mathrm{~ms}$ [Figs. 4(a) and 4(b1)]. As described previously for sensory responses in visual cortex ${ }^{7}$ and barrel cortex ${ }^{12}$ of newborn rats, the LFP and MUA responses in S1 and M1 consist of an early and a late component [Fig. 4(a1)]. As in our VSDI (cf. Fig. 3), the average amplitude of the evoked response is larger in $\mathrm{S} 1 \quad(\sim 2.8 \mathrm{mV})$ compared with $\mathrm{M} 1 \quad(\sim 1.9 \mathrm{mV})$ [Fig. 4(b2)]. Furthermore, the response is longer in S1 ( 1.4 s) compared with M1 ( 1 s) [Fig. 4(b3)]. These data demonstrate that sensory stimulation in newborn animals induces two spatially distinct local responses in S1 and M1 and that immature M1 functions as motor as well as somatosensory cortex.

Interactions between $\mathrm{S} 1$ and cortical motor areas have been previously documented in adult rodents. ${ }^{30,60-63}$ Sensory information can reach M1 either by thalamic projections to M1 or by associative connections from $\mathrm{S} 1$ (for a review, see Refs. 62 and 64). Simultaneous electrophysiological recordings of spontaneous neocortical activity and spontaneous forepaw movements in newborn rats demonstrate that $\sim 40 \%$ of the spontaneous gamma and spindle bursts in M1 are triggered by forepaw movements. About $36 \%$ of the spontaneous activity in
M1 is not correlated with any movements. Only about onefourth $(\sim 24 \%)$ of the forepaw movements are preceded by both gamma and spindle bursts in M1 followed by network activity in $\mathrm{S} 1,{ }^{35}$ indicating that these "spontaneous" forepaw movements are triggered by M1. Accordingly, electrical microstimulation of L5 in M1 in the typical frequency of spindle bursts and gamma oscillations reliably elicits forepaw movements, and local inactivation of M1 reduces forepaw movements. ${ }^{35}$ These observations suggest that a motor-sensory loop contributes to gamma oscillations and spindle bursts in M1 of newborn rodents, as has been previously suggested by Khazipov et al. ${ }^{9}$ Coordinated motor behavior can not only be observed in newborn rodents (for a review, see Ref. 65), but also in preterm human babies, ${ }^{15,66}$ indicating functional interactions between the somatosensory and motor systems during early developmental stages.

It is currently not completely understood where and how the spontaneous activity patterns in sensory neocortical areas of newborn rodents and preterm human infants are generated. In this respect, several brain structures are potential candidates to fulfill the role of a central pattern generator (CPG) (for a review, see Ref. 1). Spontaneous whisker twitches may be generated by CPGs located in the spinal cord (for a review, see Ref. 67), the brainstem (for a review, see Ref. 68), or 
M1 ${ }^{69,70}$ However, these studies have been performed in adult animals, and it remains to be elucidated which brain structure and network act as a CPG in the newborn rodent.

\section{Conclusions}

Experimental studies and clinical findings strongly suggest that spontaneous network activity during early development plays an important role in the functional maturation of sensory cortices and the development of functional topography maps. ${ }^{54,56,71}$ Our VSDI and MEA data indicate that spontaneous as well as sensory-evoked gamma oscillations and spindle bursts in M1 and S1 may contribute to the generation of the topographic and columnar organization within the somatosensory-motor system.

In addition to this instructive role of early synchronized activity in establishing the cortical architecture, it has also been demonstrated that spontaneous activity controls the process of naturally occurring programmed cell death (apoptosis) in the developing cerebral cortex. Blockade of spontaneous network activity in developing neocortical cultures causes a $\sim 2.5$-fold increase in the number of apoptotic neurons. ${ }^{72}$ More recent data indicate that physiologically relevant burst patterns control apoptosis, ${ }^{73}$ whereas pathological activity patterns interfere with programmed cell death. ${ }^{74}$ Distinct survival-promoting pathways are activated differently depending on the type of electrical activity, ${ }^{75}$ indicating that the synchronized activity patterns are particularly suited to control apoptosis in the developing cerebral cortex.

It is of pivotal interest to gain a better understanding how drugs (e.g., ethanol and nicotine) or medication (e.g., anesthetics and anti-epileptics) taken by the pregnant mother may reach the fetus by crossing the placenta and may disturb activity patterns in the developing brain. ${ }^{76}$ Drugs generally given to the preterm infant such as caffeine or theophylline to treat apnea of prematurity may also have severe effects on early network activity. ${ }^{77}$ Experimental studies combining MEA recordings with VSDI in the developing brain in vivo may be most helpful to understand the functional consequences of these pathophysiological activity patterns from a cellular level to large-scale cortical interactions.

\section{Acknowledgments}

This work was supported by grants of the Deutsche Forschungsgemeinschaft to the author. I am most thankful to former and present coworkers for many helpful discussions and for their contributions to "Barrel Cortex Function." All experiments described in this review were performed in accordance with the national laws for the use of animals in research and approved by the local ethical committee (\#23177-07/G10$1-010)$.

\section{References}

1. H. J. Luhmann et al., "Spontaneous neuronal activity in developing neocortical networks: from single cells to large-scale interactions," Front. Neural Circuits 10, 40 (2016).

2. R. Khazipov and H. J. Luhmann, "Early patterns of electrical activity in the developing cerebral cortex of human and rodents," Trends Neurosci. 29, 414-418 (2006).

3. C. Allene and R. Cossart, "Early NMDA receptor-driven waves of activity in the developing neocortex: physiological or pathological network oscillations?" J. Physiol. 588, 83-91 (2010).

4. J. W. Yang et al., "Spindle bursts in neonatal rat cerebral cortex," Neural Plast. 2016, 1 (2016).
5. R. Khazipov, M. Minlebaev, and G. Valeeva, "Early gamma oscillations," Neuroscience 250, 240-252 (2013).

6. I. L. Hanganu, Y. Ben-Ari, and R. Khazipov, "Retinal waves trigger spindle bursts in the neonatal rat visual cortex," J. Neurosci. 26, 6728-6736 (2006).

7. M. T. Colonnese et al., "A conserved switch in sensory processing prepares developing neocortex for vision," Neuron 67, 480-498 (2010).

8. M. Minlebaev, Y. Ben-Ari, and R. Khazipov, "Network mechanisms of spindle-burst oscillations in the neonatal rat barrel cortex in vivo," J. Neurophysiol. 97, 692-700 (2007).

9. R. Khazipov et al., "Early motor activity drives spindle bursts in the developing somatosensory cortex," Nature 432, 758-761 (2004).

10. J. W. Yang et al., "Three patterns of oscillatory activity differentially synchronize developing neocortical networks in vivo," J. Neurosci. 29, 9011-9025 (2009).

11. M. Minlebaev et al., "Early gamma oscillations synchronize developing thalamus and cortex," Science 334, 226-229 (2011).

12. J. W. Yang et al., "Thalamic network oscillations synchronize ontogenetic columns in the newborn rat barrel cortex," Cereb. Cortex 23, 1299-1316 (2013).

13. S. Vanhatalo and K. Kaila, "Development of neonatal EEG activity: from phenomenology to physiology," Semin. Fetal Neonat. Med. 11, 471-478 (2006).

14. M. Chipaux et al., "Auditory stimuli mimicking ambient sounds drive temporal 'delta-brushes' in premature infants," PLoS One 8, e79028 (2013).

15. M. Milh et al., "Rapid cortical oscillations and early motor activity in premature human neonate," Cereb. Cortex 17, 1582-1594 (2007).

16. E. A. Tolner et al., "Subplate neurons promote spindle bursts and thalamocortical patterning in the neonatal rat somatosensory cortex," J. Neurosci. 32, 692-702 (2012).

17. M. J. Benders et al., "Early brain activity relates to subsequent brain growth in premature infants," Cereb. Cortex 25, 3014-3024 (2015).

18. K. K. Iyer et al., "Cortical burst dynamics predict clinical outcome early in extremely preterm infants," Brain 138, 2206-2218 (2015).

19. A. Grinvald et al., "Voltage-sensitive dye imaging of neocortical activity," Cold Spring Harbor Protoc. 2016 (2016).

20. A. Grinvald et al., "Imaging the neocortex functional architecture using multiple intrinsic signals: implications for hemodynamic-based functional imaging," Cold Spring Harbor Protoc. 2016 (2016).

21. A. Grinvald and R. Hildesheim, "VSDI: a new era in functional imaging of cortical dynamics," Nat. Rev. Neurosci. 5, 874-885 (2004).

22. A. Grinvald and C. C. Petersen, "Imaging the dynamics of neocortical population activity in behaving and freely moving mammals," Adv. Exp. Med. Biol. 859, 273-296 (2015).

23. A. Grinvald et al., "Imaging the dynamics of mammalian neocortical population activity in-vivo," Adv. Exp. Med. Biol. 859, 243-271 (2015).

24. J. C. Jung et al., "In vivo mammalian brain imaging using one- and twophoton fluorescence microendoscopy," J. Neurophysiol. 92, 3121-3133 (2004).

25. S. L. Resendez et al., "Visualization of cortical, subcortical and deep brain neural circuit dynamics during naturalistic mammalian behavior with head-mounted microscopes and chronically implanted lenses," Nat. Protoc. 11, 566-597 (2016).

26. V. Reyes-Puerta et al., "Propagation of spontaneous slow-wave activity across columns and layers of the adult rat barrel cortex in vivo," Brain Struct. Funct. 1-21 (2016) (in press).

27. V. Reyes-Puerta et al., "Laminar and columnar structure of sensoryevoked multineuronal spike sequences in adult rat barrel cortex in vivo," Cereb. Cortex 25, 2001-2021 (2015).

28. S. An et al., "Long-term potentiation in the neonatal rat barrel cortex in vivo," J. Neurosci. 32, 9511-9516 (2012).

29. M. H. Mohajerani et al., "Mirrored bilateral slow-wave cortical activity within local circuits revealed by fast bihemispheric voltage-sensitive dye imaging in anesthetized and awake mice," J. Neurosci. 30, 3745-3751 (2010).

30. I. Ferezou et al., "Spatiotemporal dynamics of cortical sensorimotor integration in behaving mice," Neuron 56, 907-923 (2007).

31. A. Hernandez et al., "Procedure for recording the simultaneous activity of single neurons distributed across cortical areas during sensory discrimination," Proc. Natl. Acad. Sci. U. S. A. 105, 16785-16790 (2008). 
32. A. Luczak et al., "Sequential structure of neocortical spontaneous activity in vivo," Proc. Natl. Acad. Sci. U. S. A. 104, 347-352 (2007).

33. D. R. Kipke et al., "Advanced neurotechnologies for chronic neural interfaces: new horizons and clinical opportunities," J. Neurosci. 28, $11830-11838$ (2008).

34. E. H. Ratzlaff and A. Grinvald, "A tandem-lens epifluorescence macroscope: hundred-fold brightness advantage for wide-field imaging," J. Neurosci. Methods 36, 127-137 (1991).

35. S. An, W. Kilb, and H. J. Luhmann, "Sensory-evoked and spontaneous gamma and spindle bursts in neonatal rat motor cortex," J. Neurosci. 34, 10870-10883 (2014).

36. O. Mitrukhina et al., "Imprecise whisker map in the neonatal rat barrel cortex," Cereb. Cortex 25, 3458-3467 (2015).

37. A. J. Borgdorff, J. F. Poulet, and C. C. Petersen, "Facilitating sensory responses in developing mouse somatosensory barrel cortex," J. Neurophysiol. 97, 2992-3003 (2007).

38. C. C. Petersen, A. Grinvald, and B. Sakmann, "Spatiotemporal dynamics of sensory responses in layer $2 / 3$ of rat barrel cortex measured in vivo by voltage-sensitive dye imaging combined with whole-cell voltage recordings and neuron reconstructions," J. Neurosci. 23, 1298-1309 (2003).

39. I. Ferezou, S. Bolea, and C. C. Petersen, "Visualizing the cortical representation of whisker touch: voltage-sensitive dye imaging in freely moving mice," Neuron 50, 617-629 (2006).

40. P. Golshani et al., "Internally mediated developmental desynchronization of neocortical network activity," J. Neurosci. 29, 10890-10899 (2009).

41. M. Kummer et al., "Column-like $\mathrm{Ca}(2+)$ clusters in the mouse neonatal neocortex revealed by three-dimensional two-photon $\mathrm{Ca}(2+)$ imaging in vivo," Neuroimage 138, 64-75 (2016).

42. K. Kirmse et al., "GABA depolarizes immature neurons and inhibits network activity in the neonatal neocortex in vivo," Nat. Commun. 6 , 7750 (2015)

43. J. C. Crowley and L. C. Katz, "Early development of ocular dominance columns," Science 290, 1321-1324 (2000).

44. H. J. Luhmann, M. L. Martinez, and W. Singer, "Development of horizontal intrinsic connections in cat striate cortex," Exp. Brain Res. 63, 443-448 (1986).

45. P. Rakic, "Specification of cerebral cortical areas," Science 241, 170176 (1988)

46. P. Rakic et al., "Decision by division: making cortical maps," Trends Neurosci. 32, 291-301 (2009).

47. E. Friauf, S. K. McConnell, and C. J. Shatz, "Functional synaptic circuits in the subplate during fetal and early postnatal development of cat visual cortex," J. Neurosci. 10, 2601-2613 (1990).

48. I. L. Hanganu, W. Kilb, and H. J. Luhmann, "Functional synaptic projections onto subplate neurons in neonatal rat somatosensory cortex," J. Neurosci. 22, 7165-7176 (2002)

49. S. Hirsch and H. J. Luhmann, "Pathway-specificity in N-methyl-daspartate receptor-mediated synaptic inputs onto subplate neurons," Neuroscience 153, 1092-1102 (2008)

50. P. O. Kanold and H. J. Luhmann, "The subplate and early cortical circuits," Апnи. Rev. Neurosci. 33, 23-48 (2010).

51. I. L. Hanganu et al., "Cellular mechanisms of subplate-driven and cholinergic input-dependent network activity in the neonatal rat somatosensory cortex," Cereb. Cortex 19, 89-105 (2009).

52. H. J. Luhmann, W. Kilb, and I. L. Hanganu-Opatz, "Subplate cells: amplifiers of neuronal activity in the developing cerebral cortex," Front. Neuroanat. 3, 19 (2009).

53. E. Dupont et al., "Rapid developmental switch in the mechanisms driving early cortical columnar networks," Nature 439, 79-83 (2006).

54. W. Kilb, S. Kirischuk, and H. J. Luhmann, "Electrical activity patterns and the functional maturation of the neocortex," Eur. J. Neurosci. 34, 1677-1686 (2011).
55. M. T. Colonnese and R. Khazipov, "Slow activity transients in infant rat visual cortex: a spreading synchronous oscillation patterned by retinal waves," J. Neurosci. 30, 4325-4337 (2010).

56. L. A. Kirkby et al., "A role for correlated spontaneous activity in the assembly of neural circuits," Neuron 80, 1129-1144 (2013).

57. V. Sreenivasan et al., "Parallel pathways from motor and somatosensory cortex for controlling whisker movements in mice," Eur. J. Neurosci. 41, 354-367 (2015).

58. T. V. Gerdjikov et al., "Rhythmic whisking area (RW) in rat primary motor cortex: an internal monitor of movement-related signals?" J. Neurosci. 33, 14193-14204 (2013).

59. C. C. H. Petersen, "Cortical control of whisker movement," Annu. Rev. Neurosci. 37, 183-203 (2014).

60. F. Matyas et al., "Motor control by sensory cortex," Science 330, 12401243 (2010)

61. T. Y. Mao et al., "Long-range neuronal circuits underlying the interaction between sensory and motor cortex," Neuron 72, 111-123 (2011).

62. R. Aronoff et al., "Long-range connectivity of mouse primary somatosensory barrel cortex," Eur. J. Neurosci. 31, 2221-2233 (2010).

63. J. L. Chen et al., "Behaviour-dependent recruitment of long-range projection neurons in somatosensory cortex," Nature 499, 336-340 (2013).

64. D. Feldmeyer et al., "Barrel cortex function," Prog. Neurobiol. 103, 3-27 (2013).

65. F. Clarac, F. Brocard, and L. Vinay, "The maturation of locomotor networks," Prog. Brain Res. 143, 57-66 (2004).

66. G. Cioni and H. F. Prechtl, "Preterm and early postterm motor behaviour in low-risk premature infants," Early Hum. Dev. 23, 159-191 (1990).

67. W. J. Moody and M. M. Bosma, "Ion channel development, spontaneous activity, and activity-dependent development in nerve and muscle cells," Physiol. Rev. 85, 883-941 (2005).

68. Y. Nakamura and N. Katakura, "Generation of masticatory rhythm in the brain-stem," Neurosci. Res. 23, 1-19 (1995).

69. A. M. Hattox, C. A. Priest, and A. Keller, "Functional circuitry involved in the regulation of whisker movements," J. Comp. Neurol. 442, 266276 (2002).

70. F. Haiss and C. Schwarz, "Spatial segregation of different modes of movement control in the whisker representation of rat primary motor cortex," J. Neurosci. 25, 1579-1587 (2005).

71. P. Rahkonen et al., "Cortical somatosensory processing measured by magnetoencephalography predicts neurodevelopment in extremely low-gestational-age infants," Pediatr. Res. 73, 763-771 (2013).

72. N. Heck et al., "Activity-dependent regulation of neuronal apoptosis in neonatal mouse cerebral cortex," Cereb. Cortex 18, 1335-1349 (2008).

73. A. Golbs et al., "Control of programmed cell death by distinct electrical activity patterns," Cereb. Cortex 21, 1192-1202 (2011).

74. B. Nimmervoll et al., "LPS-induced microglial secretion of TNF-alpha increases activity-dependent neuronal apoptosis in neonatal cerebral cortex," Cereb. Cortex 23, 1742-1755 (2013).

75. S. Papadia et al., "Synaptic NMDA receptor activity boosts intrinsic antioxidant defenses," Nat. Neurosci. 11, 476-487 (2008).

76. J. Lebedeva et al., "Inhibition of cortical activity and apoptosis caused by ethanol in neonatal rats in vivo," Cereb. Cortex (2015) (in press).

77. A. Ruangkittisakul et al., "Methylxanthine-evoked perturbation of spontaneous and evoked activities in isolated newborn rat hippocampal networks," Neuroscience 301, 106-120 (2015).

Heiko J. Luhmann studied biology at the University of Bremen and did his PhD at the Max-Planck-Institute for Brain Research in Frankfurt. After a postdoc period at Stanford University he worked as research associate at the University of Cologne and assistant professor at the Charité. In 1995 he became associate professor at the University of Duesseldorf and in 2002 full professor of physiology at the University Medical Mainz. He is interested in cortical development. 